Signal \& Image Processing : An International Journal (SIPIJ) Vol.4, No.2, April 2013

\title{
WAVELET PACKET BASED IRIS TEXTURE ANALYSIS FOR PERSON AUTHENTICATION
}

\author{
Sunita Lokhande ${ }^{1}$ and Vishram . N. Bapat ${ }^{2}$ \\ ${ }^{1}$ Sinhgad College of Engineering Pune , Maharashrta \\ sunitalokhande@yahoo.co.in \\ ${ }^{2}$ Ganga Institute of Techonology And management, Kablana \\ Haryana, NCR, Delhi \\ vbkanji@gmail.com
}

\begin{abstract}
There is considerable rise in the research of iris recognition system over a period of time. Most of the researchers has been focused on the development of new iris pre-processing and recognition algorithms for good quail iris images. In this paper, iris recognition system using Haar wavelet packet is presented. Wavelet Packet Transform (WPT) which is extension of discrete wavelet transform has multi-resolution approach. In this iris information is encoded based on energy of wavelet packets.. Our proposed work significantly decreases the error rate in recognition of noisy images. A comparison of this work with nonorthogonal Gabor wavelets method is done. Computational complexity of our work is also less as compared to Gabor wavelets method.
\end{abstract}

\section{KEYWORDS}

Biometrics, Iris recognition, Iris segmentation, Iris normalization, Wavelet packet.

\section{INTRODUCTION}

Automated person identification/verification based on biometrics has been received extensive attention due to an increasing importance of security. The two categories of biometric identifiers include physiological and behavioral characteristics. Physiological characteristics are those which a person physically owns like fingerprints, iris patterns, face, ear, shape, hand geometry, retina patterns, palm prints etc. Behavioral characteristics are the attitude of a person like gait, voice, signature etc.

Applications of these systems include computer systems security, $e$-banking, credit card, access to buildings in a secure way. Many biometric researchers have used iris features for highly secured identification, and this has led to the extensive studies in developing iris recognition techniques [1] [2]. Iris recognition is one of the most trustworthy method compared to other biometrics due to rich and unique textures of the iris, non-invasiveness, stability of iris pattern throughout the human life time, public acceptance, and availability of user friendly capturing devices[3] [4]. Iris recognition is recognizing a person by analyzing random pattern of iris. The function of an iris recognition system is to extract, represent and compare the textural patterns of iris. Typical iris recognition system includes steps like iris capture, image pre-processing, feature DOI : 10.5121/sipij.2013.4208 
Signal \& Image Processing : An International Journal (SIPIJ) Vol.4, No.2, April 2013

extraction and matching. Iris pre-processing include localization, image enhancement and normalization.

Current iris recognition systems require that the subjects stand close to the camera and look for a period of time (cooperative image) to capture the eye image . It restricts its use where subjects' cooperation is not expectable e.g. criminal, terrorists (non- cooperative condition). Also highly heterogeneous lighting environments like natural light leads to the appearance of specular reflection in image. In this work cooperative as well non-cooperative iris images are referred. Iris localization detects the inner and outer boundaries of the iris. Eyelids and eyelashes that may cover iris are removed. Iris normalization converts iris image from Cartesian coordinates to Polar coordinators. This work propose a new iris recognition method that uses wavelet packets decomposition using Haar wavelet. Appropriate Packet energies are used to compute iris codes. For the classification of iris recognition system metrics Manhattan distance is used.

\section{EXISTING METHODS}

John Daugman [1] is the inventor of the most successful and currently commercial iris recognition system and his algorithm is based on the iris codes. Integro-differential operators are used to detect the centre and diameter of the iris. The image is converted from Cartesian coordinates to polar coordinates and the rectangular representation of the region of the interest is generated. Feature extraction algorithm uses the 2D Gabor wavelets to generate the iris codes which are then matched using Hamming distance.

Wildes [2] used an isotropic band-pass decomposition derived from application of Laplacian of Gaussian filters to the image data. Like Daugman, Wildes also used first derivative of image intensity to find the location of edges corresponding to the border of the iris. Wildes's system explicitly models the upper and lower eyelids with parabolic arcs whereas; Daugman excludes the lower and upper portions of the image. The results of the systems were good enough to recognize the individuals in minimum time period.

Boashash and Boles [8]proposed a new approach based on zero crossing wavelet transform. They first localized and normalized the iris by using edge detection and other well known computer vision algorithms. The zero crossings of the wavelet transform are then calculated at various resolution levels over concentric circles on the iris. The resulting one dimensional (1D) signals are then compared with the model features using different dissimilarity functions.

Zhu et. al. used Gabor filters and 2D wavelet transform for feature extraction. For identification , weighted Euclidean distance classification has been used. This method is invariant to translation and rotation and tolerant to illumination. The classification rate using Gabor is $98.3 \%$ and accuracy with wavelet is $82.51 \%$. L. Ma [11] used vertical, horizontal projections and Hough transform for localization followed by dyadic wavelet for feature vector generation.

M. Vatsa et. Al [19] present a novel iris verification algorithm which uses textural and topological features of the iris image. The proposed 1D log Gabor wavelet is used to extract the textural information and Euler numbers are used to extract the topological information from the iris image. They used Hamming distance algorithm and proposed difference matching algorithm to match the textural and topological information. Based on this algorithm, matching strategy is presented to reduce the false rejection while false acceptance is unaffected. 
Signal \& Image Processing : An International Journal (SIPIJ) Vol.4, No.2, April 2013

\section{PROPOSED METHOD}

Figure 1 shows the flow of the proposed method. It consists of various stages like segmentation normalization, feature extraction and matching.

\subsection{Iris Image Preprocessing}

An images from CASIA and UBIRIS database are taken as input from which iris is detected. Preprocessing of iris operates in various stages like segmentation, normalization.

\subsubsection{Segmentation}

Eye image contains many pixel regions related to sclera, eyelids outside of the iris region which do not define the pattern of interest. Therefore, the first stage in iris recognition is to separate iris boundaries from the rest part of input eye images.Various techniques are used by researchers like integro-differential operator, Hough transform, active counter method etc. Based on optimum performance [20][21], in this work, Hough transform is used to detect pupil and iris boundaries. This involves first employing Canny edge detection to generate edge boundaries [1]. The Canny Edge Detector is one of the most commonly used image processing tools, detecting edges in a very robust manner. The Canny edge detection algorithm is known to many as the optimal edge detector. Also eyelids, eyelashes and specular reflection areas if any, need to isolate. Eyelids can be isolated by using linear Hough transform. Threshold can be employed for isolating eyelashes and reflections. Figure 2 shows original eye image, the segmented Iris respectively. 
Signal \& Image Processing : An International Journal (SIPIJ) Vol.4, No.2, April 2013

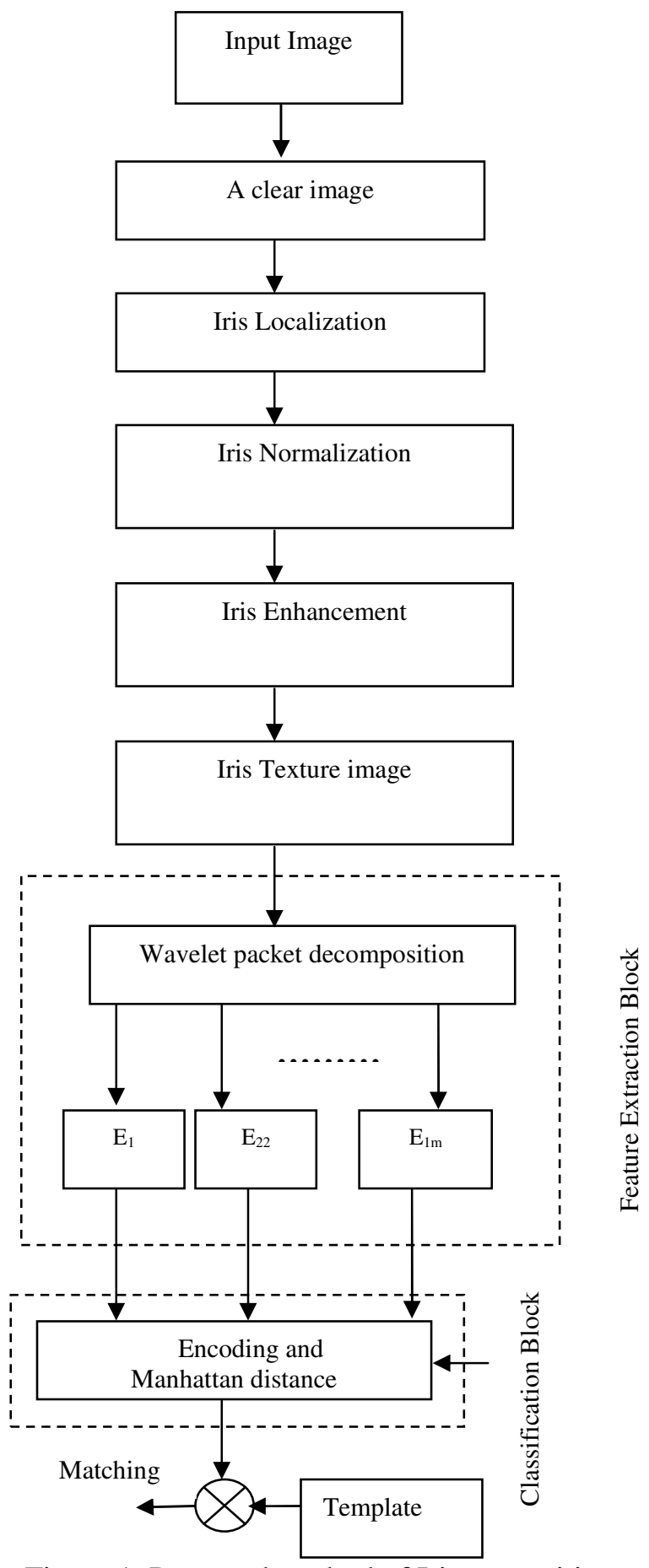

Figure 1. Proposed method of Iris recognition 
Signal \& Image Processing : An International Journal (SIPIJ) Vol.4, No.2, April 2013
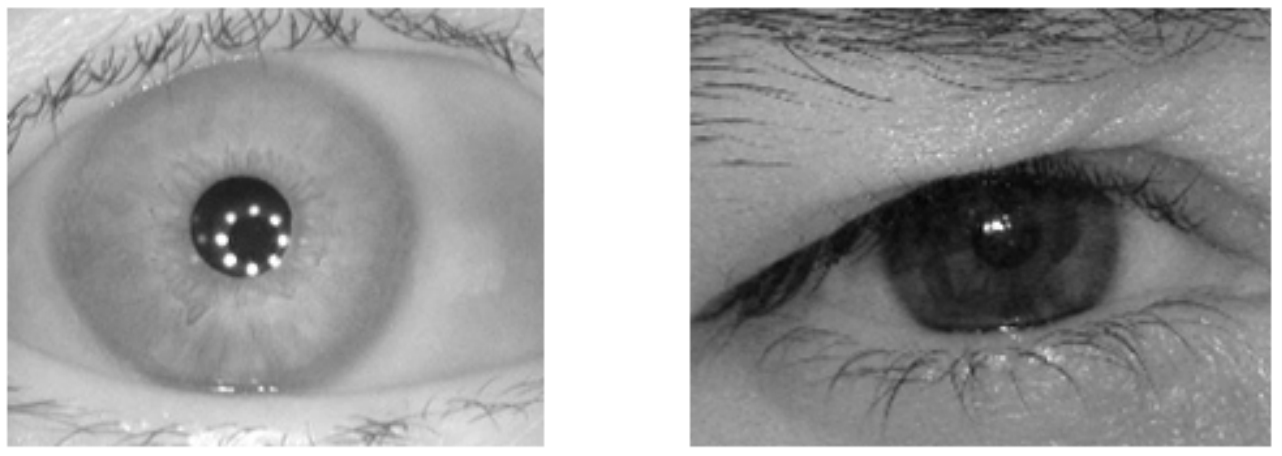

a) original eye (CASIA and UBIRIS)
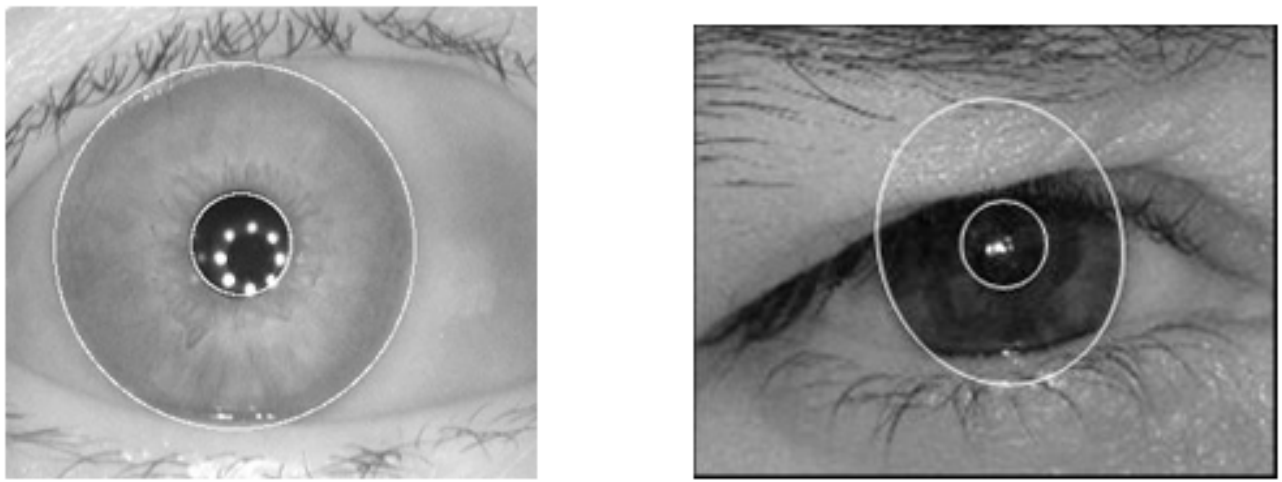

b)Segmented Iris (CASIA and UBIRIS)

Figure 2. Segmentation of iris from eye image

\section{Hough Transform}

The radius and centre coordinates of the pupil and iris regions are decided using circular Hough transform. In this, an edge map is generated by calculating the first derivatives of intensity values in an eye image and then thresholding the result. From the edge map, circles are drawn with radius $r$ and increment all coordinators that the perimeter of the circle passes through in the accumulator. Find one or more maxima in the accumulator. Map the found parameters radius, centre coordinators. The range of radius values to search for was set manually, depending on the database used. For the CASIA database, the iris radius range from 90 to 150 pixels, while the pupil radius ranges from 28 to 75 pixels.

For the more efficient and accurate circle detection process, the Hough transform for the iris/sclera boundary was performed first. Then the iris/pupil boundary is localized using the Hough transform instead of the whole eye region, since the pupil is always within the iris region. After this process six parameters are stored, the radius, $\mathrm{x}$ and $\mathrm{y}$ centre coordinates for iris and pupil circles. Upper and lower eyelids are isolated by first fitting a line using the linear Hough transform. A second horizontal line is then drawn, which intersects with the first line at the iris edge that is closest to the pupil [16]. 
Signal \& Image Processing : An International Journal (SIPIJ) Vol.4, No.2, April 2013

\subsubsection{Iris Normalisation}

The iris code matching task is performed by pairing iriscodes extracted from the query iris image and template of iris images. Iris of different people may be of different size. For the same person, the size may vary because of changes in the illumination and other factors. So, normalization is performed to get all the images in a standard form suitable for the processing. It is carried by Daugman's Rubber sheet model [1] using Cartesian co-ordinate system into polar co-ordinate system. The remapping of the iris region from $(\mathrm{x}, \mathrm{y})$ Cartesian coordinates to the polar coordinates is shown in figure 3 and figure 4 shows the result after normalization of iris with its size of $50 \times 250$ pixels.

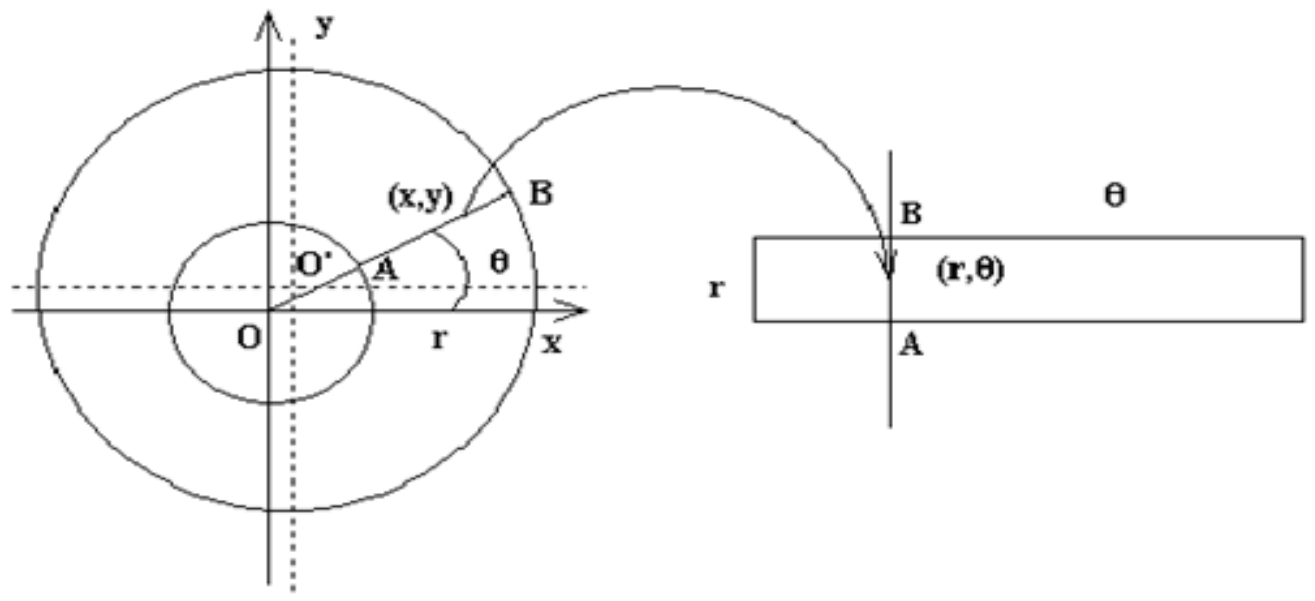

a) Cartesian coordinates

b) Polar Coordinates

Figure .3 Remapping of Iris region

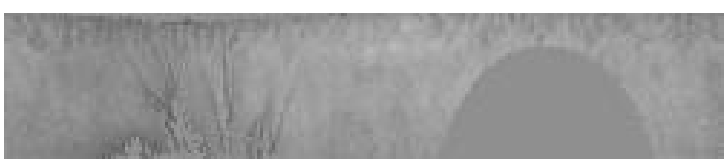

Figure 4. Iris after normalization

Non-concentric polar representation is modeled as,

$\mathrm{I}(\mathrm{x}(\mathrm{r}, \theta), \mathrm{y}(\mathrm{r}, \theta))$------- I(r, $\theta)$

With

$$
\begin{aligned}
& x(r, \theta)=(1-r) x_{p}(\theta)+r x_{i}(\theta) \\
& y(r, \theta)=(1-r) y_{p}(\theta)+r y_{i}(\theta)
\end{aligned}
$$

where, $\mathrm{I}(\mathrm{x}, \mathrm{y})=$ iris image

$(\mathrm{x}, \mathrm{y})=$ original cartesian coordinates

$(\mathrm{r}, \theta)=$ normalised coordinates 
$\left(\mathrm{x}_{\mathrm{p}}, \mathrm{y}_{\mathrm{p}}\right)=$ pupil coordinates

$\left(\mathrm{x}_{\mathrm{i}}, \mathrm{y}_{\mathrm{i}}\right)=$ iris coordinates along $\theta$ direction

\subsection{Feature extraction and encoding}

The iris has particular interesting structure and provides abundant texture information. The whole image of the iris conveys the information even at small scales. The packets method permits to process the image at each level of resolution. The feature processing task consists of feature extraction and feature encoding.

\subsubsection{Feature Extraction}

It is crucial to choose suitable method for feature extraction. Wavelet transform is faster as compared to the conventional signal processing algorithm based on fourier transform [16]. It is a technique of analyzing signals in multiresolution mode. Wavelet packet analysis is an extension of discrete wavelet transform (DWT) and it turns out that the DWT is only one of the much possible decomposition that could be performed on the image. In the wavelet packet it is possible to combine the different levels of decomposition in order to achieve the optimum time frequency representation of the image. Also using wavelet, image denoising can achieve by soft thresholding. In this work, Haar wavelet decomposition of the normalized iris is carried out up to 3- level. The Haar wavelet has compact support in time; however, it has 1 vanishing moment. It is orthonormal, piecewise constant and is defined by

$$
\psi(x)=\left\{\begin{array}{l}
1 \quad 0 \leq x<1 / 2 \\
-1 \quad 1 / 2 \leq x,<1, \\
0, \text { otherwise }
\end{array}\right.
$$

Figure 5 shows wavelet packet decomposition tree up to level 3, which is used in this work. The steps followed for feature extraction of normalized iris are as follows.

Step 1

Wavelet packet decomposition: Wavelet packet decomposition of $50 \times 250$ size normalized iris image is carried out up to level 3 using Haar wavelet. This generates 64 packet subimages each of size $7 \times 32$. In figure $5,(0,0)$ denotes the normalized iris image, $(2,0)$ denotes the $1^{\text {st }}$ packet subimage at the second level decomposition of the normalized iris and so on.

Step 2

Wavelet packet energy : A energy (E) based criterion describes information related properties for accurate representation of texture information in packet subimages and computed as below [5].

$$
E=\sum_{x} \sum_{y}|f(x, y)|^{2}
$$


$\mathrm{f}(\mathrm{x}, \mathrm{y})$ indicates iris pixel at position $(\mathrm{x}, \mathrm{y})$.

where, $x=1$ to $M, y=1$ to $N, M=$ No. of rows, N=No. of columns of normalized iris.

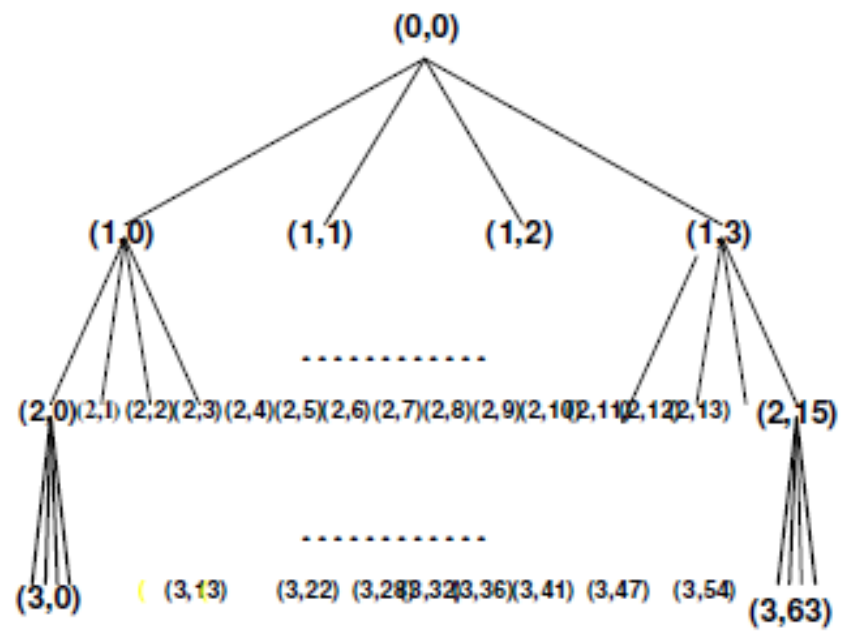

Figure 5. Wavelet Packet Decomposition tree

Figure 6 shows mean energy distribution i. e. energy $V_{s}$ packet number, only for 63 wavelet packets and packet $(3,0)$ which represent DC component is not considered. In this graph instead of packet $(3,1)$, packet number is used. e.g. Packet $(3,1)$ has packet no.1, Packet $(3,2)$ has no. 2 and so on From this graph appropriate dominant energies are chosen to compute iris code. In the figure 6 wavelet packets $(3,1),(3,2),(3,5),(3,6)$ are appropriate packet energies. Experiment results also shows that in most of images appropriate energy packets corresponds to the same packets number. The value of soft threshold (T) is computed using equation (2), which is more practical in engineering applications [11] [18].

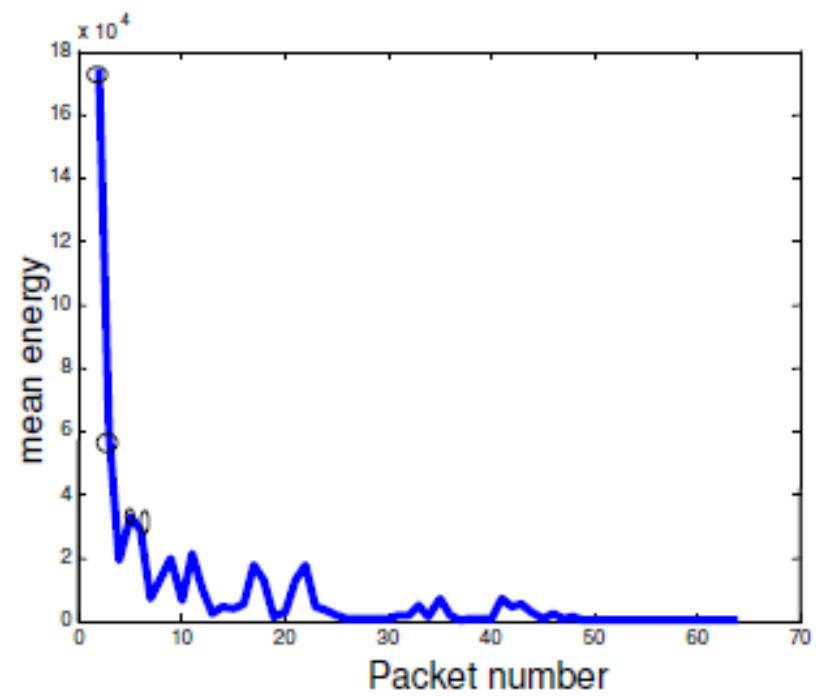

Figure 6 Mean energy distribution for iris S1061R (CASIA) 
Signal \& Image Processing : An International Journal (SIPIJ) Vol.4, No.2, April 2013

$$
\mathrm{T}=3 \sigma
$$

Where, $\sigma$ is the standard deviation of the highest frequency packet coefficients. In our case highest frequency Packet no. $(3,63)$ is used for computing threshold. Table 1 and table 2 shows first three appropriate packets of sub images for five iris images of the CASIA database person and UBIRIS database person.

Table 1. The three appropriate energy packets for iris S1061R (CASIA database) at 3-level

\begin{tabular}{|l|c|c|c|c|c|}
\hline Iris & $\mathbf{1}$ & $\mathbf{2}$ & $\mathbf{3}$ & $\mathbf{4}$ & $\mathbf{5}$ \\
\hline Packet & 3 & 2 & 3 & 3 & 3 \\
\cline { 2 - 6 } Number & 2 & 3 & 2 & 2 & 2 \\
\cline { 2 - 6 } & 5 & 5 & 5 & 5 & 5 \\
\hline
\end{tabular}

\subsubsection{Feature encoding}

After the determination of the threshold (T) the feature vectors are generated by coding the coefficients of appropriate energy packets. Let $\mathrm{E}_{\mathrm{L}}$ be the appropriate energy of $\mathrm{L}^{\text {th }}$ packet. Then

Table 2. The three appropriate energy packets for iris Img_54_2_1(UBIRIS database) at 3-level

\begin{tabular}{|l|c|c|c|c|c|}
\hline \multirow{2}{*}{ Iris } & 1 & $\mathbf{2}$ & $\mathbf{3}$ & $\mathbf{4}$ & $\mathbf{5}$ \\
\hline $\begin{array}{l}\text { Packet } \\
\text { Number }\end{array}$ & 3 & 2 & 3 & 2 & 2 \\
\cline { 2 - 6 } & 2 & 3 & 2 & 3 & 3 \\
\cline { 2 - 6 } & 5 & 5 & 5 & 5 & 5 \\
\hline
\end{tabular}

iris code $f_{L}(x)$ for this packet is computed according to equation (3)

$$
\left.\begin{array}{rl}
\mathrm{f}_{\mathrm{L}}(\mathrm{x}) \quad & \text { for } \mathrm{C}_{(\mathrm{i}, \mathrm{j}) \mathrm{L}}>\mathrm{T} \\
& =-1 \quad \text { for } \mathrm{C}_{(\mathrm{i}, \mathrm{j}) \mathrm{L}}<-\mathrm{T} \\
& =0 \quad \text { for }\left|\mathrm{C}_{(\mathrm{i}, \mathrm{j}) \mathrm{L}}\right|>\mathrm{T}
\end{array}\right\}
$$

Where, $\mathrm{C}_{(\mathrm{i}, \mathrm{j}) \mathrm{L}}$ is the $(\mathrm{i}, \mathrm{j})$ th coefficient of $\mathrm{L}^{\text {th }}$ packet .

In this work iris codes are generated using single appropriate packet and combination of appropriate packets. For single packet iris code length is 224 , for combination of two packets code length becomes 448 and so on.

\section{IRIS MATCHING}

The iris code matching task is performed by pairing iris codes extracted from the query image and template of iris images. The common metric for comparison of iris are Hamming distance, Manhattan distance (MD) etc. In this work, feature vectors are compared using a Manhattan distance. 
Signal \& Image Processing : An International Journal (SIPIJ) Vol.4, No.2, April 2013

The Manhattan distance (MD) is calculated as follows:

$$
\mathrm{MD}=\frac{1}{N} \sum_{j=0}^{N}|I(j)-T(j)|
$$

Where $\mathrm{I}$ is iris code for query image, $\mathrm{T}$ is the iris code from template and $\mathrm{N}$ is the bit length of iris code. Ideally, the distance between two images of the same iris will be 0 corresponds to genuine score and that between two images of different irises will be high which is impostor score [3].

\section{EXPERIMENTAL RESULTS}

There are several public and freely available iris image database for biometric purpose. To evaluate the proposed algorithm, our approach used CASIA and UBIRIS iris database. CASIA images incorporate few types of noise like eyelid and eyelashes obstruction. UBIRIS database include more noisy images. Proposed approach is compared with best known algorithm like Daugman 2 D Gabor filter and Boles method.

Experiment results are represented in terms of false acceptance rate (FAR) and false rejection rate (FRR). Receiver Operating Curve (ROC) curve is plotted to measure accuracy for equal error rate (EER). At EER both FRR and FAR are equal. FAR and FRR are calculated.

$$
\begin{aligned}
\text { FAR } & =\frac{\sum_{0}^{\mathrm{k}} \mathrm{MD}}{\sum_{0}^{\max } \mathrm{MD}} \\
\mathrm{FRR} & =\frac{\sum_{\mathrm{k}}^{\max } \mathrm{MD}}{\sum_{0}^{\max } \mathrm{MD}}
\end{aligned}
$$

Where $\mathrm{k}$ is the separation point between intra-class and inter-class. The experiment is carried out on five different set of iris codes generated from quantizing appropriate packets using equation 3 . Then performance is evaluated for all sets to investigate the optimum code length. In this, first iris code set is generated by using only $(3,1)$ packet, second iris code from only $(3,2)$ packet, third by only $(3,4)$ packet then remaining sets by combination of these packets as shown in table 3 . We test the proposed algorithm on both data sets.

\section{i)Using CASIA database}

Figure 7 shows Receiver operating characteristics curve (ROC) for different bit length and it is a graph between FAR and FRR. From this curve EER for different set code are calculated. Smaller the EER better is performance. Table 3 shows the EER for different bit length. With increasing bit length EER decreases, but with little bit improvement in accuracy after 448 bit length. Combining too many packets doesn't improve the performance much as compare to large bit length .Combination of $2^{\text {nd }}$ and $3^{\text {rd }}$ appropriate energy packets gives optimum performance with accuracy $97 \%$. 
Signal \& Image Processing : An International Journal (SIPIJ) Vol.4, No.2, April 2013

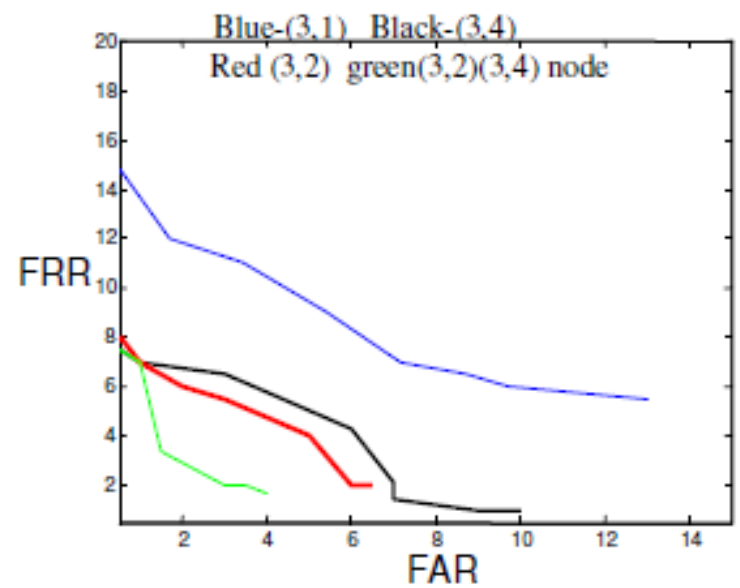

Figure 7 ROC curve for different bit length.

Table 3 EER for different bit length of code (CASIA- database)

\begin{tabular}{|l|l|c|}
\hline Appropriate packets at node & $\begin{array}{l}\text { Iris code } \\
\text { length }\end{array}$ & EER (\%) \\
\hline$(3,1)$ & 224 & 7.17 \\
\hline$(3,2)$ & 224 & 6.02 \\
\hline$(3,4)$ & 224 & 5.50 \\
\hline$(3,10)$ & 224 & 8.25 \\
\hline$(3,2)$ and $(3,4)$ & 448 & 3.36 \\
\hline$(3,2)$ and $(3,1)$ & 448 & 4.04 \\
\hline$(3,2),(3,4)$ and $(3,1)$ & 672 & 3.17 \\
\hline$(3,2),(3,4)(3,1)$ and $(3,10)$ & 896 & 3.02 \\
\hline
\end{tabular}

\section{ii) Using UBIRIS-database}

Performance is also evaluated for noisy UBIRIS database. From previous experiment study, in this case iris code is generated for only three sets as shown in table 4 . In this case optimum value of EER is $8.73 \%$.

Experiment conducted over CASIA and UBIRIS shows that matching accuracy is not increasing rapidly with combination of the iris code. Therefore, $(3,2)(3,4)$ are considered as a optimum packet combination for comparison with existing system. Table 5 and table 6 shows comparison with existing system in CASIA and UBIRIS respectively. 
Signal \& Image Processing : An International Journal (SIPIJ) Vol.4, No.2, April 2013

Table 4 EER for different bit length of code (UBIRIS- database)

\begin{tabular}{|l|c|c|}
\hline $\begin{array}{c}\text { Appropriate packets } \\
\text { at node }\end{array}$ & $\begin{array}{c}\text { Iris code } \\
\text { length }\end{array}$ & EER (\%) \\
\hline$(3,2)$ and $(3,4)$ & 448 & 8.73 \\
\hline$(3,2)$ and $(3,1)$ & 448 & 11.04 \\
\hline$(3,2),(3,4)$ and $(3,1)$ & 672 & 8.25 \\
\hline
\end{tabular}

Table 5 Comparison With existing methods (CASIA)

\begin{tabular}{|c|c|c|}
\hline Methods & CRR (\%) & EER(\%) \\
\hline 2DGabor filter & 99.88 & 0.08 \\
\hline Boles & 92.64 & 8.13 \\
\hline Proposed & 96.64 & 3.36 \\
\hline
\end{tabular}

Table 6 Comparison With existing methods (UBIRIS)

\begin{tabular}{|c|c|c|}
\hline Methods & CRR (\%) & EER(\%) \\
\hline 2D Gabor Filter & 89.24 & 10.34 \\
\hline Proposed & 91 & 8.73 \\
\hline
\end{tabular}

\section{Conclusions}

In this paper, we have addressed cooperative and non-cooperative iris recognition method. For less noisy CASIA database 2-D Gabor filter perform better due to its shift invariant property. But its performance degraded in noisy images. In the present work, we have observed the significant impact of noise in the accuracy of iris recognition algorithm. Therefore, we proposed new iris recognition strategy more robust to noise. In the proposed method iris information is encoded based on soft thresholding which is helpful for denoising. Our proposed method did not outperform in less noisy image (CASIA) but its degradation is minor in the noisy database than 2-D Gabor filter. This shows our method is more suitable for the application within non cooperative images.

The dimensionality of the feature vector in 2-D Gabor wavelet method is higher (2048) while proposed method has smaller bit length only 448 with comparable accuracy. Proposed method is effective and robust. 
Signal \& Image Processing : An International Journal (SIPIJ) Vol.4, No.2, April 2013

\section{REFERENCES}

[1] John Daugman, "How iris recognition works" IEEE Transaction on Circuits and Systems for Video Technology, Volume no. 1,PP.21-30, 2004

[2] Richard P. Wildes, "Iris recognition :An Emerging Biometric Technology ," Proc. IEEE ,Vol. 85, PP 1348-1363, 1997

[3] John Daugman, "Biometric Personal Identification System based on Iris Analysis " United State Patent , no. $5291560,1994$.

[4] Daubenchies, "The Wavelet Transform, time frequency localization and Signal Analysis ," IEEE Transaction Information Theory, Vol. 36, PP. 961-1005, 1990

[5] Arivazhagan S. and Ganesan L. "Texture Classification Using Wavelet Transform , " Pattern recognition letters ,24, 1513-1521, 2003

[6] Users M., "Texture Classification and Segmentation using wavelet frames", IEEE Transaction on image processing, 4(11), 1995 PP. 1549-1560.

[7] Turkoglu I., Arslan A, and Ilkay E, “ An intelligent System for Diagnosis of Heart Value diseases with Wavelet Packet Neural Networks" Computer in Biology and Medicine .33(4), 319-331.

[8] Boles and Boashash, "A Human Identification Technique Using Images of the Iris and the Wavelet Transform", IEEE Transactions on Signal Processing, 1998.

[9] Li Ma, Tieniu Tan, Yunhong Wang, and Dexin Zhang, "Efficient Iris Recognition by Characterizing Key Local Variations", IEEE Transactions on Image Processing, Vol 13, No. 6, June 2004.

[10] Tisse et al., "Person Identification Technique using Human Iris Recognition", Proc. of Vision Interface, 2002.

[11] Li Ma, Tieniu Tan, Yunhong Wang, and Dexin Zhang, "Personal Identification Based on Iris Texture Analysis", IEEE Transactions on Pattern Analysis and Machine Intelligence,Vol. 25, No. 12, December 2003.

[12] D.Field, "Relations between the statistics of natural images and response properties of cortical cells", Journal of optical society of America, 1987.

[13] A. Basit, M. Y. Javed, M. A. Anjum, "Efficient Iris Recognition Method for Human Identification", World Academy of Science, Engineering and Technology 4, 2005.

[14] Abdualkadir Sengur, Ibrahim Turkoglu and M. Cevdet Ince Expert systems with Applications 32 (2007) 527-533. Wavelet packet neural networks for texture classification, ScienceDirect Expert systems with Applications 32 (2007) 527-533.

[15] A. Abhyankar and S. Schuckers, "A novel biorthogonal wavelet network system for off-angle iris recognition," Pattern Recognit., vol. 43, pp. 987-1007, 2010.

[16] S. Mallat and W. Hwang. Singularity detection and processing with wavelets IEEE transaction on information theory , 38(2):617-643,1992

[17] Rossant F. Eslava M.T. Ea T. Aml F. Amara A. , "Iris Identification and robustness Evaluation of wavelet packets based algorithm" IEEE International conference on Image processing vol.3 pp. .III 257-260

[18] Maarten Jansen, "Noise Reduction by Wavelet Thresholding," Springer -Verlag New York Inc.2001.

[19] M. Vatsa, R. Singh, and A. Noore, "Reducing the false rejection rate of iris recognition using textural and topological features," Int. J. Signal Process., vol. 2, no. 1, pp. 66-72, 2005.

[20] Minal Pawer and S.S. Lokhande "Comparison of iris segmentation methods for performance enhancement of recognition "CIIT international journal ,June 2012.

[21] Minal Pawer and S.S. Lokhande "Iris segmentation using Geodesic Active ontur for improved texture extraction in recognition" IJCA ,. International Journal of Computer Applications (0975 888) Volume 47- No.16, June 2012 
Signal \& Image Processing : An International Journal (SIPIJ) Vol.4, No.2, April 2013

\section{Authors}

Sunita Shashikant Lokhande received the B.E. (Electronics) and M.E. (Electronics) degrees from Shivaji University, Kolhapur, Maharashtra State, India, in 1992 and 2006 respectively.

Presently she is working as a Associate Professor in the Department of Electronics and Telecommunication Engineering at Sinhgad College of Engineering, Pune, India. Her research interests include digital signal processing, image processing, filter banks, applications of wavelet transform, and biometrics.

Vishram Nilkanth Bapat received the B.E. (Electrical) and M.E.(Electrical) degrees from Shivaji University, Kolhapur, Maharashtra State, India. He received Ph. D. in Electrical Engineering from Indian Institute of Technology, Kharagpur. Presently he is working as a Director at Ganga Institute of Technology \& Management, Bahadurgarh, Delhi. His research interests include digital signal processing, image processing, Communication networks and biometrics.
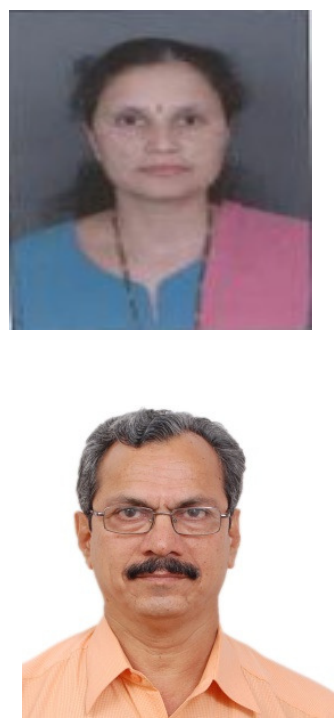